\title{
Review and Preview: Disocclusion by Inpainting for Image-based Rendering
}

\author{
Zinovi Tauber, Ze-Nian Li, Mark S.Drew
}

\begin{abstract}
Image-based rendering takes as input multiple images of an object and generates photorealistic images from novel viewpoints. This approach avoids explicitly modeling scenes by replacing the modeling phase with an object reconstruction phase. Reconstruction is achieved in two possible ways: recovering 3D point locations using multiview stereo techniques, or reasoning about consistency of each voxel in a discretized object volume space. The most challenging problem for image-based reconstruction is the presence of occlusions. Occlusions make reconstruction ambiguous for object parts not visible in any input image. These parts must be reconstructed in a visually acceptable way. This paper both reviews image inpainting and argues that inpainting can provide not only attractive reconstruction but as well a framework increasing the accuracy of depth recovery.

Digital image inpainting refers to any methods that fillin holes of arbitrary topology in images so that they seem to be part of the original image. Available methods are broadly classified as structural inpainting or textural inpainting. Structural inpainting reconstructs using prior assumptions and boundary conditions, while textural inpainting only considers available data from texture exemplars or other templates. Of particular interest is research of structural inpainting applied to 3D models, emphasizing its effectiveness for disocclusion.
\end{abstract}

Index Terms-Disocclusion, Image Inpainting, Imagebased Rendering, Depth from Stereo, Volumetric Reconstruction, View-Dependent Rendering.

\section{INTRODUCTION}

\section{A. Motivation}

Photorealistic rendering is a computer process that generates images indistinguishable from photographs. It is one of the main objectives of computer graphics. When scene realism is the main concern, the easiest way to achieve this is by photographing or filming the desired scene. It is often desired to incorporate into a scene objects that would be difficult to acquire on film, either due to physical limitations, or practical limitations such as positioning of cameras. These objects can be modeled on a computer by an artist, but this process is very laborious and rarely achieves realistic imagery. Illumination, complex shapes, materials and interaction dynamics are all very difficult to model in a realistic way. On the other hand, it is difficult to reproject photographs onto virtual cameras in space. The two main problems are that the depths of the visible scene points are unknown, and that nothing is known of the occluded points. Disocclusion is the attempt of recovering scene information obstructed by visible parts.

Realistic computer models can be obtained using 3D acquisition methods on existing objects or models (maquettes). Acquisition using images is versatile and yields more

Z. Tauber is with the Department of Computing Science in Simon Fraser University, British Columbia, Canada. detail than other 3D acquisition methods. Unfortunately, image correspondences for multiview stereo matching are hard to accomplish reliably. Most stereo reconstruction approaches initially establish epipolar geometry between a reference view and any additional views. The epipolar constraint for a pixel in the reference view indicates that its line of sight (LOS) ray projects to an epipolar line in another view image (see [1] [2] for stereo correspondence reviews). For dense matching, a disparity map is calculated for all pixels in the reference view by matching them to the pixels on the corresponding epipolar lines of the second view. There are many issues that complicate matching, the worst being occlusions - in the presence of occlusions some pixels will have no corresponding pixels [3].

Image-based rendering techniques combine both vision and graphics processes to reconstruct an object from multiple images, and reproject it to a novel view. The ability of these methods to handle occlusions, despite many innovations, is insufficient and could benefit greatly from integration with principles from digital inpainting.

For any number of cameras, reconstruction algorithms might face a family of shapes to choose from [4], all projecting identically to all camera images due to occlusions. In order to estimate the occluded object sections we need prior knowledge or assumptions about the model. Disocclusion algorithms have been studied in computer vision for purposes such as segmentation or stereo matching [5] [6]. Recently, such techniques have taken a new role, that of restoration of images with occluded and damaged regions, called holes, where the location of these regions is known. Bertalmio et al. [7] have formulated the problem in terms of a diffusion of image structure elements from the hole boundary into the hole. This process was called digital image inpainting, a term borrowed from the arts used to describe a restoration process for damaged paintings. Research in the image inpainting field focuses on improving assumptions for connectivity of object structures in the hole, as well as inpainting of texture based on statistical or template data. Image inpainting has also been performed in a rudimentary fashion on surfaces in 3D with holes due to occlusions. This paper argues that inpainting methodology, extended to 3D, is an essential step for multi-view object reconstruction. We propose a framework wherein an object surface is the structure component, with a displacement map applied as the texture component. This will enable rendering object sections that otherwise cannot be reconstructed. Moreover, this framework can be used to improve disparity matching in $3 \mathrm{D}$ reconstruction. 


\section{B. Overview of Surveyed Literature}

Reconstruction tasks such as image restoration, object disocclusion, texture segmentation and synthesis, and surface extraction share some similar underlying principles. These problems admit a probabilistic model in which each possible state of each element is assigned some probability drawn from a random field, most commonly a Markov Random Field (MRF) [8][9]. Then, reconstructing an image is accomplished by finding the Maximum A-Posteriori (MAP) hypothesis. Gibbs fields can calculate the equivalent MRF probability; they explicitly depend on an energy functional, which can be more easily minimized than the probability itself in order to find the MAP hypothesis. New energy functionals can be constructed that are driven by some envisioned process, rather than by explicitly modeling the likelihood of states.

Early work on disocclusion was done by Nitzberg, Mumford and Shiota [6]. In their work they attempted to generate outlines of objects for image segmentation and depth extraction. T-junctions were detected in an edge map, and corresponding $\mathrm{T}$-junctions were connected using an energy functional minimizing edge length and curvature. Masnou and Morel [10] extended the idea to level-sets of images. In this way, all the gray-levels of the occluded object can be overpainted. In an inspiring paper, Bertalmio et al. [7] proposed digital image inpainting. With a manually selected inpainting region $\Omega$ in an image, the objective of inpainting is to complete the image inside the hole in a reasonable way. Their approach was to propagate the gray levels on the hole boundary $\partial \Omega$ into the hole along isophotes (level lines), by formulating the inpainting as a variational problem and solving by diffusion. This diffusion coupled with anisotropic filtering was shown to have an interpretation as fluid transportation using the Navier-Stokes equations of fluid dynamics [11], an approach which helps with stability and speed of convergence.

Inpainting methods that involve more complicated energy functionals assume the Bounded Variation ( $B V)$ image model [10][12][13][14]. This model states that image level-lines cannot oscillate infinitely, and immediately suggests a simple Total Variation ( $T V$ ) inpainting technique, which tries to minimize the curve length. However, it was discovered that better reconstructions are achieved when the inpainting considers curvature as well. Ballester and Bertalmio et al. [12] re-cast the inpainting process to include curvature, in a form similar to the Euler elastica. Chan and Shen [14] defined a new inpainting called Curvature Driven Diffusion (CDD), and in a later paper remarkably showed how the Euler elastica encapsulate both CCD inpainting and transportation inpainting [13].

Many additional types of inpaintings were proposed subsequently, including textural inpainting [15]-[18] which relies on texture matching and replication, or global image statistics [19], or templates matching functionals [20]. Finally, research was also done on inpainting in 3D, by explicitly reconstructing surfaces [21] or by applying the inpainting suggested in [7] to generate a surface in a volume grid $[22]$.
The earliest research on image-based rendering used image warping techniques to generate new views of a realistic or a computer generated scene. Chen and Williams [23] extended the idea to 3D by calculating a linear warp field between corresponding 3D points of two scenes, and interpolated for views in between. Their research tries to deal with both holes and visibility ordering. The Lightfield [24] and Lumigraph [25] provide a more accurate and complete capability of viewing from any point in the sampled space. The space is sampled regularly, and a 4D lattice of images is created. Any single view direction corresponds to a $2 \mathrm{D}$ slice in the space, interpolated from nearest neighbors as necessary. Acquisition, data storage, and access are main concerns here. In Plenoptic Modelling, McMillan and Bishop [26] described a complete framework for generating new views from arbitrary viewpoints. First, cylindrical image samples are generated, and then a form of stereo matching is performed on the cylinders for dense correspondence.

Image-based rendering methods can be classified into two types of approaches: those that are based on multiview stereo correspondences and generate a single depth map [3][26]-[31], and volumetric reconstructions which store multiple depths per pixel, and reason about voxel visibilities using consistency checks with input images [32][4][33][34]. Some methods make improvements in quality and realism by using view dependent optimizations. View dependent texture mapping is performed by most methods that texture map image information [29][27][35]. These methods emphasize the effectiveness of $2 \mathrm{D}$ texture when a simplified 3D shape is known, and lead us to suggest inpainting of $3 D$ structure and texture as a more complete and photorealistic solution.

In Section II we present the evolution of image restoration and inpainting towards a more unified variational problem. Other forms of inpainting methods are presented as well. Section III briefly presents some representative techniques in image-based rendering and argues for the necessity of the inpainting methodology. Section IV provides a short summary and conclusions. An expanded version of this article is available in [36].

\section{Disocclusion And Digital InPainting}

\section{A. Disocclusion and Image Restoration}

The problems of disocclusion and boundary continuation can be viewed as a particular area of image restoration. Most work in image restoration focuses on de-noising, enhancing colors, and recovering isolated missing pixels. Yet approaches that disocclude objects have also been attempted. Still, traditional formulations for filling in holes are limited in scope to regions where some information is known in a close proximity, and so cannot handle most disocclusion problems.

Bilinear and bicubic interpolations can be used to interpolate holes, and more advanced interpolations are possible as well. Adaptive anisotropic filters can have an edge preserving property (e.g. [37]). Single pass filtering have 
undesired blurring of the inpainted regions, yet most inpainting schemes are applied by repeated application of boundary preserving filters.

Modeling the image properly can help restoring data reliably. Three possible approaches are presented in [38]: I. Random Fields, most prominently MRFs, capture the statistical properties of some function $u$, such as the image function over the discrete image domain $\mathcal{S}$. II. Physical Processes in a steady state, like fluid flow, can approximate image continuity. III. Function Spaces assume characteristics about an image energy function $E(u)$. One of the most common function spaces for image inpainting is the Bounded Variations (BV) space.

MRFs are a useful modeling tool for many applications in computer vision, from image restoration to stereo matching. According to the Hammersley-Clifford theorem, an MRF of a set of random variables $U$ is a Gibbs Random Field (GRF) on $\mathcal{S}$ when the GRF takes the form:

$$
p(u)=\frac{1}{Z} e^{-\frac{1}{T} E(u)}, \quad Z=\sum_{u \in U} e^{-\frac{1}{T} E(u)} .
$$

where $T$ is the temperature, and is responsible for the variability in the distribution. $Z$ is called the partition function and normalizes the quantity $p(u)$ to lie in $[0,1]$. It is quite hard to compute $Z$ since the number of computed terms is combinatorial in the size of $\mathcal{S}$. However, for most applications $Z$ is constant and so the MAP estimate is given by simply minimizing the energy function $E(u)$. More information about modeling with MRFs is given in [8]. We can combine MRFs with MAP estimation according to Bayes theorem to obtain the MAP-MRF hypothesis: the value $u^{*}$ that maximizes the posterior probability $p\left(u \mid u_{0}\right)$ is

$$
u^{*}=\arg \max _{u} p\left(u \mid u_{0}\right)=\arg \min _{u} E\left(u_{0} \mid u\right)+E(u) .
$$

where $u$ is the ideal function we wish to recover and $u_{0}$ is the observed function.

As an example of MRFs in image reconstruction, consider the problem of interpolating the function $u \equiv u(x, y)$ on the image domain $\mathcal{S}$, from sparse data. This problem is equivalent to restoration of a depth surface obtained from sparse data in stereo matching. Assume that the observation model $E\left(u_{0} \mid u\right)$ is given by Eq. 3 below, for a white (Gaussian) noise $u_{0}=K * u+n_{G}$, with $u$ the ideal noise free image function, $u_{0}$ the observed noisy image function, $K$ a smoothing kernel, and $n_{G}$ a random value drawn from a Gaussian distribution with standard deviation $\sigma$ (* denotes convolution) [38].

$$
E\left(u_{0}, K, \sigma\right)=\frac{1}{2 \sigma^{2}|\mathcal{S}|} \int_{\mathcal{S}}\left(u_{0}-K * u\right)^{2} d x d y .
$$

Note that the white noise has a specific Gibbs distribution pattern, and so can be used here directly.

The prior model $E(u)$ is designed to maintain the continuity principle. Instead of merely penalizing large first order derivatives of $u$, a better smoothness assumption might require the second order derivative to be small as well. Additionally, the model should allow piecewise discontinuity, by composing with a function $g$ that imposes a penalty term $\gamma$ for discontinuity. A possible choice can be the truncated quadratic $g(\eta)=\min \left\{\eta^{2}, \gamma\right\}$. Thus the prior model is

$$
E(u)=\int_{\mathcal{S}}\left[g\left(u_{x x}\right)+2 g\left(u_{x y}\right)+g\left(u_{y y}\right)\right] d x d y .
$$

(the subscripts denote partial derivatives with respect to the variables.) This model is called the weak plate. Without $g$ in the formulation above, an image edge discontinuity would incur a very large penalty and the minimization would prefer to connect adjacent patches. While the minimization of the posterior $E\left(u \mid u_{0}\right)$ can be done by a gradient decent search, such a function is not strictly convex, and a gradient decent method could get stuck in a local minimum. More advanced optimization techniques are possible, both deterministic and stochastic [39].

\section{B. Structural Inpainting}

Digital image inpainting is a computer process inspired by the strokes artists use to cover up defects in paintings [40][41]. Four observations noted by Bertalmio et al. [7] guide the process: (1) The global picture determines how to fill in the hole. (2) Contours arriving at the boundary of the hole are continued inside it. (3) Different regions inside the hole are assigned similar colors to their colors at the boundary. (4) Small details are painted in.

Formally, we can define $\Omega \in \mathcal{S}$ to be an open bounded subset of $\mathcal{S}$ with Lipschitz continuous boundary, where $\mathcal{S}$ is a rectangle in $\mathbb{R}^{2} . \Omega$ is called the hole in the image $\mathcal{S}$, and terminates at a border $\partial \Omega$. We also define $\bar{\Omega}$ as the closure of $\Omega$, and $\tilde{\Omega}$ as another, surrounding, open region in $\mathcal{S}$ such that $\bar{\Omega} \subset \tilde{\Omega}$. The boundary is defined as the band $B=\tilde{\Omega} \backslash \bar{\Omega}$, consising of the pixels surrounding the hole region from which we are going to diffuse information into the hole region.

\section{B.1 Diffusion}

Inpainting approaches use either isotropic diffusion or more complex anisotropic diffusion (few algorithmic approaches do not use diffusion [42][43]). Isotropic diffusion blends information in $\Omega$ and cannot preserve image structures, and hence requires a much smaller inpainting domain. In [44], Oliveira et al. used a diffusion process involving repeated convolution of $\tilde{\Omega}$ with small isotropic smoothing kernels of size $3 \times 3$. The main advantage of such an approach over anisotropic diffusion is the high speed of calculations. Another common diffusion method, based on Euler's energy functional, is

$$
E(u)=\int_{x \in \Omega}|\nabla u|^{2} d x .
$$

As in the MRF example, this is a prior on surface smoothness. The solution to minimizing such cost functions is given by gradient descent search:

$\left.u^{0}\right|_{\Omega}=$ Some initial value: random, or simple interpolation; 
$u^{n+1}=u^{n}+\lambda \frac{\partial u^{n}}{\partial t}$

where $\lambda$ is an acceleration parameter to speed up convergence of the algorithm. Superscripts indicate the time step. In the discrete case we can approximate the energy by the equation $E(u)=\sum_{x \in \Omega}(u(x+1)-u(x))^{2}$, which has the following set of partial derivatives:

$$
\frac{\partial}{\partial t} u(x)=\frac{\partial}{\partial u(x)} E(u)=-2(u(x-1)-2 u(x)+u(x+1))
$$

which are a discretized Laplacian, as expected [45]. A more complex inpainting based on the Euler energy is found in [46], the energy defined on a curve is presented in [47], and in [48] it is adapted for highlights removal. While in general isotropic diffusion is noticeably imperfect for image intensities, it makes more sense for diffusion of orientation, because the human visual system is sensitive to contrast and sharpness changes but less so to curvature changes, which are also much less frequent. Perona has reported thoroughly on how to apply orientation diffusion in images [45]. He defines a new energy function motivated from various physical forces, which can deal with the ambiguity resulting from angle subtraction. Jiang et al. [49] proposed an extension of the idea to gray value inpainting, especially for missing DCT blocks during image transmission. Contrast changes inside the hole are hard to approximate this way. It is also possible to learn diffusion filters, as in [50] within an MRF framework.

Masnou and Morel [10] presented disocclusion using level lines of larger objects in images using variational formulations and level sets, yet still solved the problem using traditional vision techniques. The original variational continuation formulation was done earlier by Nitzberg, Mumford and Shiota [6]; however it was aimed at image segmentation, and was based on T-junctions in the edge map, which are generally few and unreliable in a natural image. Masnou and Morel extended the idea to level lines which bound upper level sets defined at each gray level $\lambda$, via

$$
X_{\lambda} u=\{x \mid x \in \mathcal{S}, u(x) \geq \lambda\}
$$

Thus the insight here is to continue each level curve into the hole left by the occluding object, rejoining the same level curve outside the hole.

\section{B.2 BV, TV}

To define more precisely the BV image model assumption that is commonly adapted, let $\mathcal{S}$ be the image domain and $u \in L^{1}(\mathcal{S})$ be a function over the domain. If $u$ 's partial derivatives at each site are measured with finite total variation in $\mathcal{S}$, then $u$ is a function of bounded variations. The class of bounded variation functions on the domain $\mathcal{S}$ is denoted by $B V(\mathcal{S})$ [51][52][12]. We can define the total variation (TV) Radon seminorm as the energy equation

$$
\begin{aligned}
E_{T V}(u) & =\int_{\mathcal{S}}|D u| d x \\
& =\sup \left\{\int_{\mathcal{S}} u \nabla \cdot \varphi d x\left|\varphi \in C_{0}^{\infty}(\mathcal{S}),\right| \varphi \mid \leq 1\right\}
\end{aligned}
$$

6) The Radon measure $|D u|=|\nabla u|$ when the gradient exists [51]. The energy $E_{T V}$ is minimized in the TV inpainting scheme [53][54]. A set $X \subset \mathcal{S}$ has a finite perimeter if its characteristic function $\chi_{X} \in B V(\mathcal{S})\left(\chi_{X}=1\right.$ when $x \in X$ and $\chi_{X}=0$ otherwise). Furthermore, the boundary length of the set $X$ when it is Lipschitz continuous is given by $\operatorname{Perim}(X)=E_{T V}\left(\chi_{X}\right)$. The TV inpainting is restated using level sets in the Coarea formula [38][52]

$$
E_{T V}(u)=\int_{-\infty}^{\infty} \operatorname{Perim}\left(X_{\lambda} u\right) d \lambda
$$

which shows that the total variation inpainting is equivalent to minimizing the length of the level lines, and results in piecewise straight lines in the inpainting domain.

Masnou and Morel propose minimizing level lines using the functional

$$
E(u)=\int_{\mathcal{S}}|D u|\left(1+\nabla \cdot \frac{D u}{|D u|}\right) d x,
$$

where as usual $\left.u\right|_{\mathcal{S} \backslash \Omega}=\left.u_{0}\right|_{\mathcal{S} \backslash \Omega}$, the observed function outside the hole. Note that the divergence $\nabla \cdot \frac{D u}{|D u|}$ is the curvature $\kappa$ of $u$. This energy can be interpreted as driving the minimization of the length of the level lines, and the angle total variation. The addition of 1 in 11 is necessary in order not to discount the length despite small change in the orientation of the line. This is a particular form of the Euler elastica discussed below. In order to minimize this equation, the T-junctions of each level line with the boundary set $\Omega$ are first detected. A dynamic programming approach can then pair up all $\mathrm{T}$-junctions in a way that minimizes the cost function in Eq. 11.

\section{B.3 PDEs}

Bertalmio and Sapiro et al. proposed inpainting using the mechanism of PDEs and diffusion [7]. The inpainting smoothly propagates the image information along the level lines directions (isophotes) from outside to inside the hole. The isophote direction, denoted by $\nabla^{\perp} u$, is normal to the gradient $\nabla u$. It is the direction of least change in gray values, a relaxation on level-lines continuity. The image information propagated is an image smoothness measure given by the Laplacian

$$
L(u)=u_{x x}+u_{y y}
$$

While the energy equation is not explicitly given in this work, the propagation of the change in image smoothness along level lines is given by the PDE

$$
\frac{\partial u}{\partial t}=\nabla L(u) \cdot \nabla^{\perp} u \text {. }
$$

This evolution only applies to $u(x)$ when $x \in \Omega$, with the boundary conditions given by $B$. We can easily see that in the steady state when $\frac{\partial u}{\partial t}=0$, the direction of the largest information change is perpendicular to the isophotes as required. The implementation requires numerical stability considerations as discussed in [55]. 

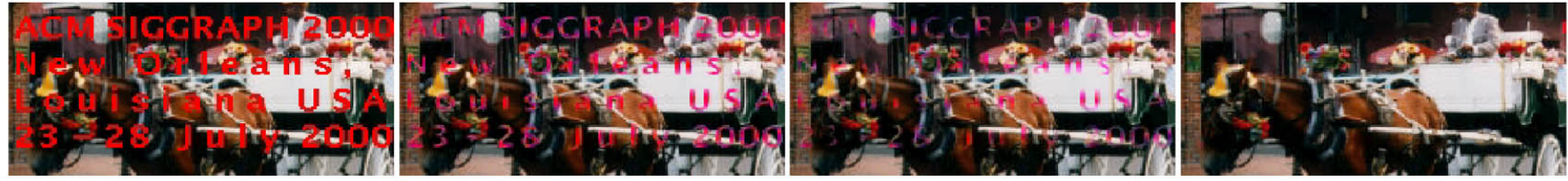

Fig. 2. Progress frames in the process of inpainting red text. (From [7]).
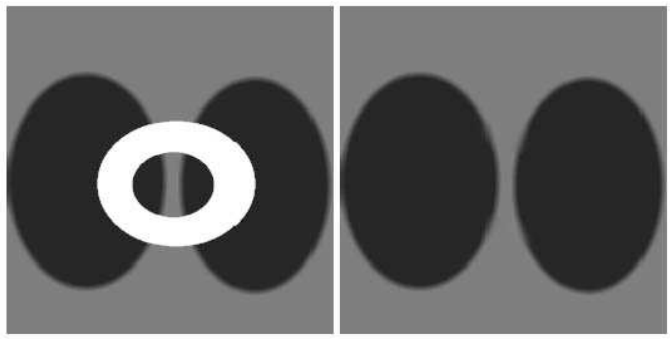

Fig. 1. Synthetic image, where $\Omega$ is the white region, and the inpainting result. (From [7]).

It is important to notice from Eq.13 that the image smoothness will continue along isophote directions in a straight line until a conflict occurs. Therefore, it is proposed that after every few iterations of inpainting, a few iterations of an anisotropic diffusion that preserves sharpness be applied. Fig. 1 shows an artificial example of two objects and an inpainting domain, showing that this method can inpaint preserving shape, and sharpness (or nearly so). However, it is possible to see loss of sharpness in the inpainting regions. Fig. 2 shows inpainting results on a real scene, with small inpainting domain.

Structure diffusion techniques have a general problem inpainting textured objects, which has been addressed with some success by textural inpainting. It follows that $3 \mathrm{D}$ textural inpainting is required for $3 \mathrm{D}$ objects as well.

\section{B.4 Fluid Flow}

The previous inpainting was shown to have an equivalent formulation using fluid dynamics [11]. The inpainting diffusion can be considered as a transport equation that convects the image intensity along level curves of the smoothness. If we define the velocity field $\boldsymbol{v}=\nabla^{\perp} u$, then $u$ is convected by the velocity field $\boldsymbol{v}$. The image model is now formulated as governed by the incompressible fluid flow Navier-Stokes equations, where we define a stream function $\Psi$ such that $\nabla^{\perp} \Psi=\boldsymbol{v}$. Additionally, the vorticity is given by $\omega=\nabla \times \boldsymbol{v}$ which in $2 \mathrm{D}$ is a scalar equal to the Laplacian $L(\Psi)$ of the stream. In the steady state, we can describe the vorticity (in the absence of viscosity) as

$$
\nabla^{\perp} \Psi \cdot \nabla L(\Psi)=0
$$

The equivalence to the image diffusion process is immediately seen: The stream function $\Psi$ is equivalent to the image function $u$, the fluid velocity $\boldsymbol{v}$ is the isophote direction $\nabla^{\perp} u$, and the vorticity $\omega$ is the same as the image smoothness $L(u)$. The viscosity $\nu$ is the anisotropic diffusion factor: Including this, we obtain a vorticity transport equation for the image

$$
\omega_{t}+\boldsymbol{v} \cdot \nabla \omega=\nu \nabla \cdot(g(|\nabla \omega|) \nabla \omega)
$$

The inpainting proceeds in a similar manner to the previous method, with similar results yet significantly reduced running time due to faster convergence and better optimization using established methods in fluid dynamics. In [56][57] a similar style of inpainting is suggested, generalized to a common vector-valued diffusion functional based on the trace of the product of the image Hessian and an anisotropic tensor, which could take the form of an oriented Laplacian.

\section{B.5 Higher Order PDEs}

More extensive formulations have been developed using higher order PDEs and the Bounded Variations image model. Let the boundary data $u_{0} \in L^{\infty}(\partial \Omega)$ and $\boldsymbol{\theta}$ be the gradient direction vector field. A variational problem can be formulated requiring the gradient field to be in the direction $\boldsymbol{\theta}$ after separate diffusions of both $u$ and $\boldsymbol{\theta}$ [12]. A relaxation on this condition can be expressed as

$$
\boldsymbol{\theta} \cdot \nabla u-|\nabla u|=0,|\boldsymbol{\theta}| \leq 1
$$

Ideally $\boldsymbol{\theta}=\frac{\nabla u}{|\nabla u|}$, but with $\boldsymbol{\theta}$ zero where the gradient is null. Minimizing the constraint as a functional, after some manipulation we obtain the energy function

$$
E(u)=\int_{\Omega}|\nabla u|+\int_{\Omega}(\nabla \cdot \boldsymbol{\theta}) \cdot u
$$

with admissible class of functions $\mathcal{A}(u)=\{u \in B V(\Omega) \mid$ $\left.|u(x)| \leq\left\|u_{0}\right\|_{\infty},\left.u\right|_{B}=\left.u_{0}\right|_{B}\right\}$. The existence of a function $u$ for such a minimization is provable, and the structure inside the hole can reconstructed. However, discontinuities in gray levels are not modeled, and worse, the vector field $\boldsymbol{\theta}$ is unknown. Therefore, we have to additionally impose a prior on the propagation of gray levels inside $\Omega$.

The Euler elastica curve is the equilibrium curve of the elasticity energy, given by

$$
\varepsilon(C)=\int_{C}\left(a+b \kappa^{2}\right) d s
$$

where $C$ is the curve of integration, $d s$ is the arc length element, and $\kappa(s)$ is the Euclidean curvature [13]. The Euler elastica model imposes regularity on the smoothness of the curves allowed. The energy minimization of such a curve is the Maximum Likelihood (ML) estimation of a geometrical walk, where the rotation each step, an exponential i.i.d. random variable, is dependent on the step size. 
Instead of connecting level curves individually, the elastica is reformulated on the domain $\Omega$ similarly to the Coarea formula. For a level curve $u=\lambda$ we have $\frac{d \lambda}{d \theta}=$ $|\nabla u|$. Then the integral

$$
\begin{aligned}
& \int_{0}^{1} \int_{C(u=\lambda)}\left(a+b \kappa^{2}\right) d s d \lambda= \\
& \int_{0}^{1} \int_{C(u=\lambda)}\left(a+b \kappa^{2}\right) d s|\nabla u| d \theta=\int_{S}\left(a+b \kappa^{2}\right)|\nabla u| d x
\end{aligned}
$$

since $d s$ is orthogonal to $d \theta$. This translation can inpaint the entire domain. Relaxing the definition of the elastica as formulated above, we can write a functional of the following form

$$
E(u)=\int_{\tilde{\Omega}}|\nabla u|\left(a+b|\nabla \cdot \boldsymbol{\theta}|^{p}\right)
$$

where $p \geq 1$ gives weight to the curvature, describing a more general p-elastica model. As in the Masnou and Morel approach, this model attempts to minimize the length plus angular variation of level sets. If we wanted to connect curves farther away using the curvature, then $b / a$ would be large. If, however, $a=0$ then the reconstruction might be ambiguous when the curvature is zero. When $b=0$ this is the TV inpainting. Chan and Shen [13] suggest that for p-elastica optimization, if $p \geq 3$ then the model blows up (i.e. $E(u) \rightarrow \infty$ ) if the function $u$ has stationary points in $\Omega$. They also advocate $p>1$ to restrict sharp curvatures. Relaxing the boundary constraints the functional is:

$$
E(u, c)=\int_{\tilde{\Omega}}\left(a+b \kappa^{2}\right)|\nabla u| d x+\frac{c}{2} \int_{\mathcal{S}}\left(u-u_{0}\right)^{2} d x .
$$

The first term is the prior model in the Bayesian view, and the second is the the data model based on the observed image values. From the Euler-Lagrange derivation it is seen that two orthogonal processes form this inpainting. One encapsulates the transport diffusion of Eq. 13, while the other is an application of Curvature Driven Diffusion (CDD) [14]. Hence the Euler elastica model remarkably contains both complementary transport and diffusion inpaintings, and so is a more complete model.

Results using this type of inpainting are shown in Fig. 3 for a portion of the Lena image. Figure 3(b) shows the inpainting on the gray levels, while $3(\mathrm{c}), 3(\mathrm{~d})$, and $3(\mathrm{e})$, show a particular level set, with inpainting with $p=1$ in $3(\mathrm{~d})$ and $p=2$ in 3(e). It can be seen that for $p=2$ the curvature is not penalized highly enough, and $p=1$ seems smoother.

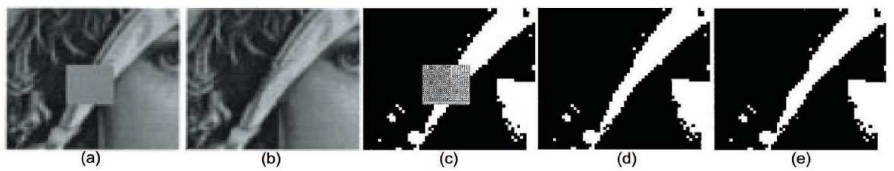

Fig. 3. Inpainting of a hole. (a) Original Lena image with hole. (b) Inpainting of the hole with $p=1$. (c) The hole on one level set. (d) Inpainting with $p=1$. (e) Inpainting with $p=2$. (From [12]).

\section{Textural Inpainting}

The inpainting models presented thus far have only used boundary data and prior assumptions about the smoothness of structures in the hole. For textured images, smoothness priors alone may not reconstruct the object faithfully, and a statistical or template knowledge of patterns inside the hole is needed as well. Modeling the statistics of an image function commonly uses MRFs. Levin et al. [19] suggest that global statistics about the image be estimated from outside the hole area, and the hole be inpainted with probability-maximizing strokes. The approach is to extract some relevant statistics about the image and then combine them in an MRF framework. A maxproduct belief propagation algorithm, which is a message passing algorithm, is used for the optimization. Since there are loops in the MRF formulation, in a large neighborhood the belief propagation gives only a local maximum [58].

A substantially different approach assumes that we have an inpainting template in another image [20]. The method has three stages: (1) Finding and matching landmark points; (2) Calculating the warp between the template and the current object; (3) Inpainting the object.

Landmarks are any feature points $p_{i}$ that can be extracted from images. After finding corresponding landmarks, the interpolation proceeds according to a thinplate spline which minimizes the bending energy of $u$ : $E=\int_{S}\left[u_{x x}^{2}+u_{x y}^{2}+u_{y y}^{2}\right] d x d y$. Solving the minimization is equivalent to interpolating with bi-harmonic radial basis functions $K(r)=r^{2} \log |r * r|$. Then, the warp $U(x)$ from the given image to the template is given by

$$
U(x)=A x+t+\sum_{i=1}^{n} w_{i} K\left(\left|x-p_{i}\right|\right),
$$

where $n$ is the number of corresponding landmarks.

Finally, to minimize intensity discontinuities, an inpainting approach is used to derive an optimal image $u$ from the image $u_{0}$ that has an inpainting domain and from a template image $u_{1}$. The inpainting energy is:

$$
a_{1} \int_{\mathcal{S}}|\nabla u|+a_{2} \int_{\mathcal{S} \backslash \Omega}\left|u-u_{0}\right|+a_{3} \int_{\Omega}\left|u-u_{1}(-U(x))\right| .
$$

The first term is the TV inpainting on the entire image domain, the second is the data term which tries to enforce boundary conditions, and the third tries to make a smooth thin-plate spline interpolation, without enforcing an exact copy.

Fig. 4 displays one result of running this algorithm, showing recovered landmarks in two different photographs: the first photograph is corrected to make the girl smile, using the whole face as an inpainting domain.

A larger number of global information techniques concern textural inpainting. A texture can be generated from examples or procedurally from statistics, though procedural methods are hard to apply for inpainting applications. The most popular texture synthesis approach for inpainting is exemplar-based synthesis. Exemplar-based 

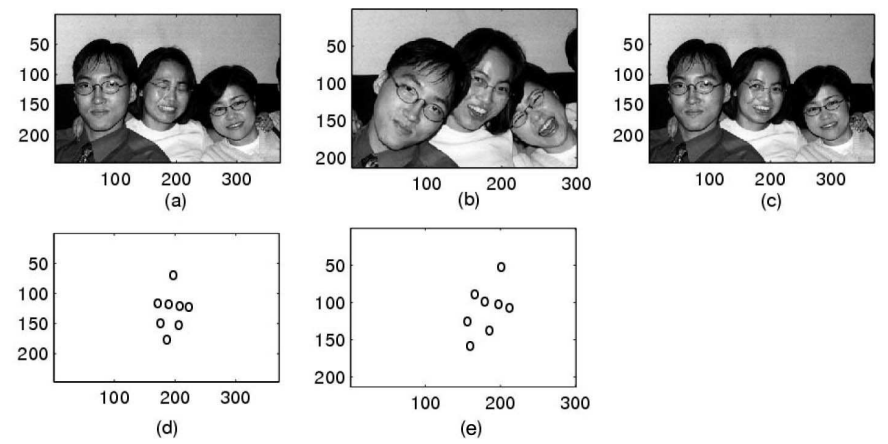

Fig. 4. Two pictures with same people in (a) and (b). In (c), the face of the middle girl in (a) was inpainted using the template image (b). (d) Landmarks for the middle girl in image (a). (e) Landmarks for the middle girl in image (b). (From [20]).

texture synthesis takes a window $\Psi(i)$ centered at a pixel $i$, and computes a Sum of Squared Differences (SSD) match with all available sample texels, simply copying the best matching patch [59].

Criminisi et al. [16] have proposed a patch exemplarbased inpainting where the inpainting pixel order depends on the structure in the image. Their insight is that the "inpainting front" should propagate along linear isophotes first, thus making the inpainting reasonable regardless of the boundary shape of the inpainting region. In contrast, a concentric filling-in of texture exemplars is sensitive to the hole boundary. Initially, pixels in $B$ are assigned a confidence value $C=1$ and pixels in $\Omega$ are assigned $C=0$. The confidence $C(i)$ and data $D(i)$ terms are defined as follows

$$
C(i)=\frac{\sum_{j \in \Psi(i) \backslash \Omega} C(j)}{\operatorname{Area}(\Psi)}, D(i)=\frac{\left|\nabla^{\perp} u(i) \cdot \nabla \partial \Omega(i)\right|}{\gamma} .
$$

The area of $\Psi$ is the patch size and $\gamma$ is a normalizing factor for intensities. The confidence term finds the average confidence of the patch, and the data term models the strength of the isophotes hitting the front $\partial \Omega$. The priority is defined as $P(i)=C(i) D(i)$ for $i$ in $\partial \Omega$. The highest priority pixel is processed first, and after a matching patch has been copied, the confidence value of the patch is applied to all copied pixels, and the front $\partial \Omega$ is recalculated along with priorities.

Fig. 5 shows an image inpainted first along stronger edges, and then in smoother regions. This approach has very convincing visual concealment if discontinuities can be avoided. A similar formulation has been used for inpainting in videos as well [18].

\section{Combined Structural and Textural Inpainting}

Criminisi et al. [16] tried to incorporate structure into textural inpainting using a very insightful principle, whereby texture is inpainted in the isophote direction according to its strength. Unfortunately this was limited to linear structures, often resulting in discontinuities where textures meet. We notice that structural inpainting tries to enforce a smoothness prior yet preserve isophotes. We can define the texture image from the decomposition

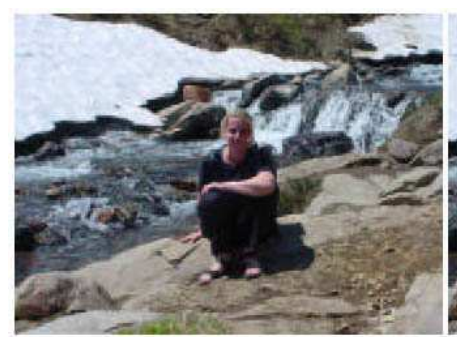

(a)

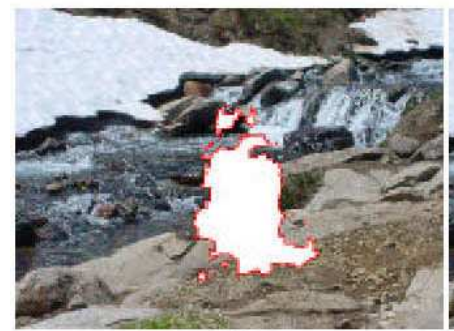

(c)

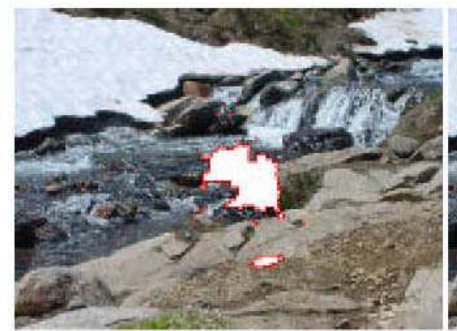

(e)

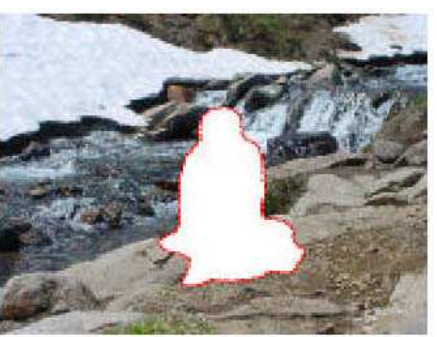

(b)

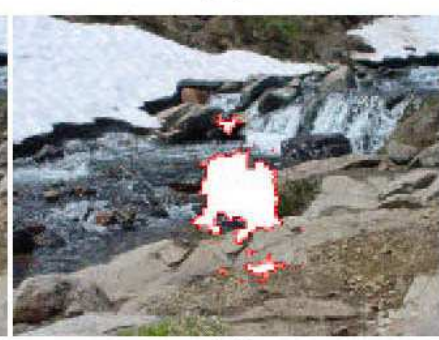

(d)

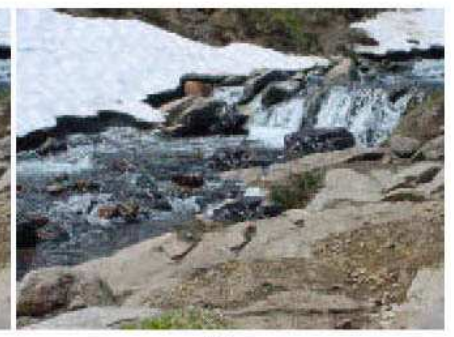

(f)
Fig. 5. Progressive stages of texture inpainting. (From [16]).

$u=u_{s}+u_{t}$, where $u_{s}$ is the structure component and $u_{t}$ is the texture component [60][15][17]. This is a redefinition of the texture in the previous subsection, which consisted of actual pixel colors. The top row of Fig. 6 shows sample results from such a decomposition. The top right image is the texture image.

Bertalmio et al. extended their inpainting to texture and structure [15] according to the decomposition above. In order to obtain the texture outside the hole they minimize the following functional:

$$
E\left(u_{s}\right)=\int_{S}\left|\nabla u_{s}\right|+\nu\left\|u-u_{s}\right\|
$$

The first term in the energy restricts the function $u_{s}$ to be in $B V(S)$, and tries to get the TV ML function $u_{s}$, while the second term tries to minimize the residual that is the texture image $u_{t}$. The balance is delicate and depends on the constant $\nu$. It is shown that if $\nu$ is small then the decoupling of texture and structure is presentable. The norm of the texture term can be defined in a new space of oscillatory functions, which is a dual of $B V$ space.

The benefit of separating texture from structure is in the ability to inpaint each sub-image separately. The texture sub-image $u_{t}$ is inpainted using an exemplar-based method that copies pixels rather than patches [61]. Note that this texture inpainting might not produce a unique solution, nor reproduce the sample characteristics faithfully. Replicating patches alleviates this problem but can introduce 
texture discontinuities as noted previously. This problem has been addressed for non-inpainting texture replication, by using a pyramid of resolutions and details [62] and by blending [63]. For the subimage $u_{s}$, inpainting proceeds as in [7] given in Eq. 13, although any other structural inpainting is applicable as well.

The results given demonstrate promising improvements over non-textural inpainting. Shown here are two results: Fig. 6 shows part of a textured image and the decomposition into texture and structure, the inpaintings of both $u_{s}$ and $u_{t}$, and the recomposition of $u$. The approach does a creditable job in continuing simple structures, yet over-smooths somewhat at the elbow, due to structure inpainting itself and ill-posed separation of structure from texture. Fig. 7 shows a second result, comparing the proposed method with the results of non-textural inpainting or texture synthesis alone. It is evident that the reconstruction is more realistic, although still noticeably flawed.

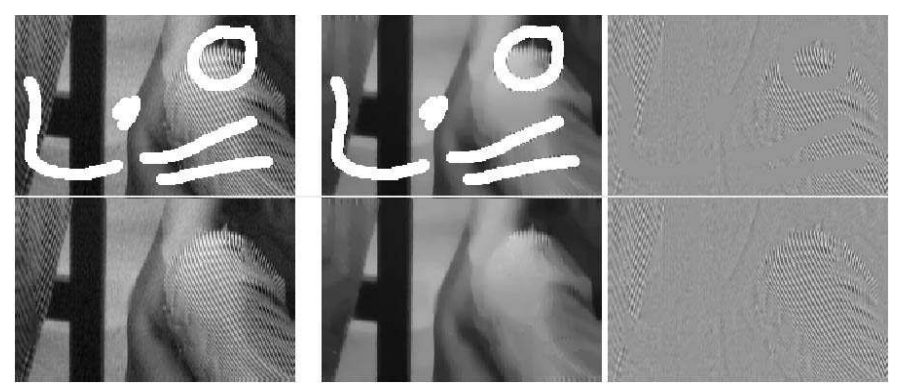

Fig. 6. Top left: Original image. Top center and right: structure and texture decomposition. Bottom left: inpainting recomposition. Bottom center and right: inpainting of structure and texture images. (From [15]).
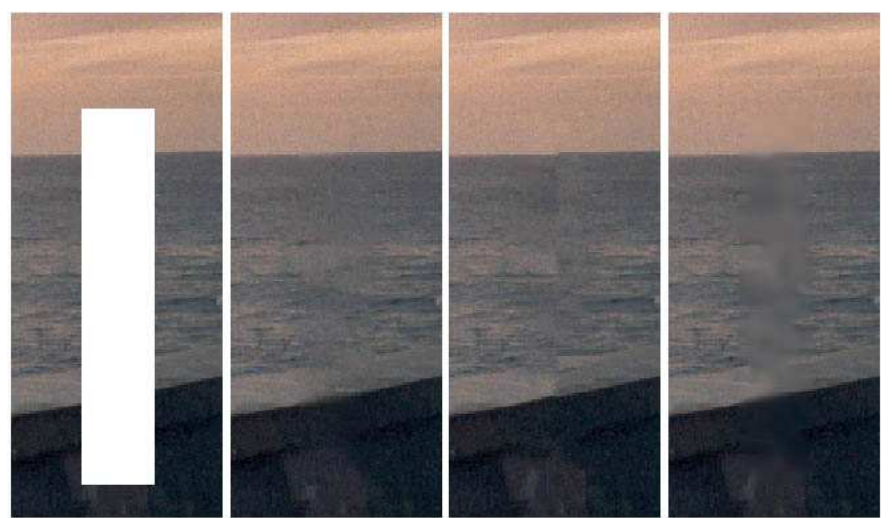

Fig. 7. From left to right: original image, inpainting using texture and structure, texture alone, and structure alone. (From [15]).

Grossauer [17] had suggested a more extensive structural and textural inpainting framework, where first the image is filtered using a curvature minimizing diffusion to generate $u_{s}$. Then $u_{s}$ is inpainted and segmented, and $u_{t}$ is inpainted using the tree-based exemplar replication algorithm due to Wei and Levoy [62]. The exemplars are taken from the same segmented region as the pixel to be inpainted, which avoids replicating mixed texture blocks. This algorithm improves upon structural and textural in- painting, but the same problems of separating texture and structure prevail. Methods also exist for structures and textures in 3D [63], but these do not support inpainting formulations.

In another segmentation approach, Jia et al. [64] proposed texture inpainting whereby the image is first texture segmented, and then region boundary curves are continued into the hole using tensor voting and B-Splines. The texture is inpainted in each region using $N \mathrm{D}$ tensor voting, for a neighborhood of size $N$, with adaptive scaling. Texture tensor voting turns out to be a very attractive method for maintaining curvature; unfortunately the method does not perform well on complex structures. Segmentation is also quite difficult to achieve correctly. However, the need for segmentation and continuation of regions generalizes into $3 \mathrm{D}$ as the need to continue surfaces separate from textures.

\section{E. Surface Inpainting}

Inpainting is potentially a very important step for imagebased rendering. While in $3 \mathrm{D}$ we can represent the data as a volume and generalize the $2 \mathrm{D}$ structural inpainting, this allows diffusion of the surface with empty space, and involves higher computational complexity than inpainting only the surface of an object.

Numerous methods exist for shape reconstruction from $3 \mathrm{D}$ points, based on implicit surfaces (e.g. [65][66][67]) or explicit surfaces (e.g. [68]), which implicitly fill in holes as well. These methods do not attempt to reconstruct the real surface shape in the hole; instead, the reconstruction has the same shape as that of the initial bounding surface. Methods have also been proposed for explicitly recovering missing surfaces by fitting parameterized curves to boundary data [21]. Since explicit surfaces are quite constrained, variational approaches are more attractive.

Verdera et al. [22] extended their image inpainting model to $3 \mathrm{D}$ using the diffusion equation Eq. 13. They redefine the 3D inpainting domain for surface reconstruction as follows: Let $A \subset R^{3}$ be a set representative of the volume spanned by the surface. Then the observed function $u_{0}$ is defined outside the hole as $u_{0}=\chi_{A}$. Define a box $Q$ around the inpainting region containing both the inpainting region and enough of $u_{0}$ to inpaint from, and define the boundary $B=Q \backslash \bar{\Omega}$ (recall that $\bar{\Omega}$ is the closure of the hole $\Omega$ ). One side of the smallest ball possible containing the inpainting region (the gap) is used to cover the gap in the surface $u_{0}$ in $Q$. This is the initial value in the inpainting region. The equivalence to $2 \mathrm{D}$ is thus established and the inpainting can proceed in a similar manner. Of note is the equivalence between gray-scale value in $2 \mathrm{D}$ and pixel location in $3 \mathrm{D}$, although in $3 \mathrm{D}$ the surface might no longer be a proper function.

Fig. 8 shows results for a laser scan of Michaelangelo's David, with occluded parts. The reconstruction appears quite successful. However, it should be noted that the gap is fairly small, and in $3 \mathrm{D}$ the problem is more difficult when the surfaces have large curvatures. This result lends credence to how effective inpainting could be for depth recovery and novel view generation in image-based rendering, 

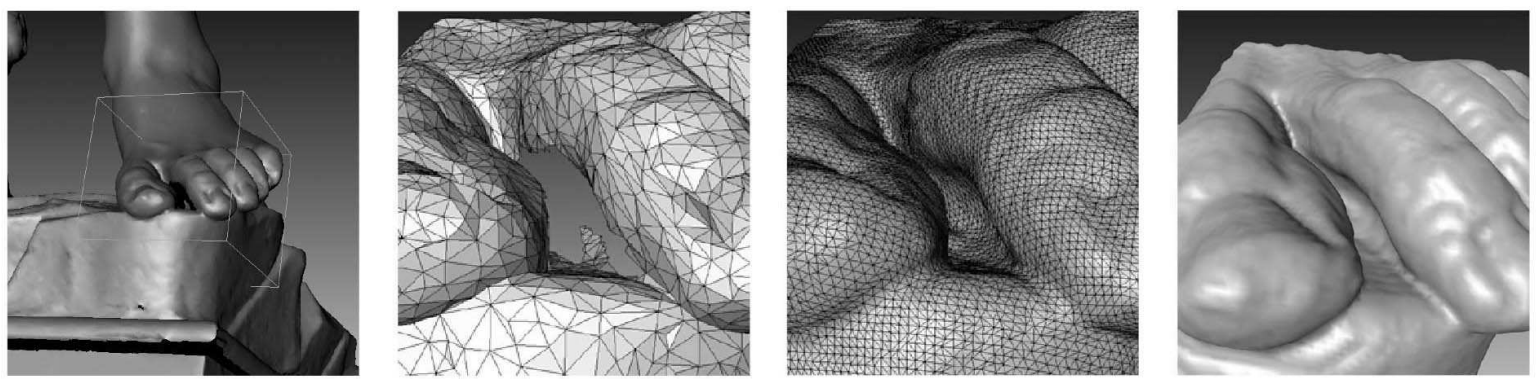

Fig. 8. The original mesh model is converted to a volume, inpainted, and then triangulated again and shaded. (From [22]).

in particular when an integrated approach is used.

Another interesting instance of inpainting in combination with surface reconstruction is given in [69]. That work attempts to reconstruct the $3 \mathrm{D}$ surface of all objects in the scene, given a single $2 \mathrm{D}$ image. The reconstruction is based on a Bayesian framework that involves prior knowledge of the type of objects, and other statistical principles such as similarity of angles for polyhedral objects and mirror symmetry. The algorithm starts with a segmentation and primal sketch of the scene, and attempts to reconstruct the $3 \mathrm{D}$ structure of objects so that their projection to the image is preserved. This approach is limited in its domain and cannot produce highly realistic images, yet it does provide many principles that inpainting could follow, and indicates what textural inpainting can accomplish in 3D.

\section{Inpainting in Image-Based Rendering}

Traditional rendering is accomplished using volume visualization techniques [70], or raster conversion techniques [71][72]. Image-based rendering is a class of various rendering approaches in which the geometrical modeling phase of traditional rendering is replaced by an image acquisition process. As a result the rendering phase can vary significantly from established rendering approaches. Initially, image-based rendering was primarily used to speed up rendering by tweening rendered frames. Today, a more important use is to acquire realistic objects that are very complex to model on a computer. This section presents a short survey of image-based rendering, emphasizing the necessity of incorporating inpainting methodology.

\section{A. View Interpolation}

The earliest techniques in image-based rendering were based on warping and morphing principles. View interpolation techniques take images as input, and use them to create novel views directly by warping without reconstruction first [23][24][25]. In contrast, newer techniques involve 3D reasoning, with a depth ordered warping frequently used for reprojection.

One of the earliest 3D view interpolations was suggested by Chen and Williams [23]. In their approach, it is assumed that all images have associated range data and the camera location in scene coordinates is known. For each pair of adjacent viewpoints, an image morph map is established in scene coordinates by taking the displacement vectors of the points in the first view transformed into the second view coordinate system. Then to render a novel view from a viewpoint on the line between the two viewpoints, the first view pixels are linearly interpolated along the morph map. Image pixels are sorted according to their depth and projected in a back-to-front order to the novel view. Visibility problems emerge, however, including many pixels contracting into one, and pixel expansion leaving gaps. To solve for ambiguity in pixel contraction, the pixels may need to be re-sorted only when the view change is larger than $\pi / 2$ degrees. When pixels expand, however, this amounts to a disocclusion problem. The filling-in algorithm they suggested was to interpolate the four corners of the pixels and then interpolate their color in the rendered image. A faster but less accurate method is actually used. The image is painted with a reserved color, and over-painted with the warped image. Gaps are detected by checking for reserved colors in the image, which are then interpolated from their neighboring pixels. A similar disocclusion problem occurs in many other image-based rendering techniques as well, including some that are based on volumetric reconstruction. The approach described above is usually employed to address this problem. Even trivially applying an inpainting algorithm to the images generated with holes will result in much improved rendering.

\section{B. Multi-view Stereo Models}

Stereo matching based techniques attempt to discover the $3 \mathrm{D}$ structure of the scene using point correspondence from multiple views of the scene. These techniques are extended and adapted for the use of image-based rendering. The basic approach of stereo matching is to calculate epipolar geometry using sparse point correspondences, and then restrict the search space to a line for recovering a depth image by dense matching [1].

Another disocclusion problem due to pixel expansion arises in Plenoptic modeling [26], which generates novel views using warp flow-fields. Using a form of multiview stereo-matching, Plenoptic samples are projected onto cylindrical manifolds. Then cylindrical epipolar geometry and dense correspondence are established between any two neighboring sample cylinders in space. Here, the McMillan depth ordering algorithm warps a source cylinder to the novel viewpoint in occlusion compatible order without depth sorting. This technique can be proven to apply to any shape of projection manifold, and is commonly used for image-based renderings that involve reprojections. This 
warping produces occlusion tears in space, and inpainting is again necessary.

We can think of multiview stereo matching as a construction of a generalized disparity space in a common coordinate frame to which all other views are transformed [28]. Finding the depth for a pixel consists of finding the best disparity value given the neighborhood and all images available (taking disparity to mean inverse depth). Generally, dense matching has the following steps: (1) For each disparity value a pixel can take, calculate its support from all available images using a matching cost such as SSD or variance. (2) Aggregate support from neighbors in disparity space, e.g. by diffusion. (3) Find the best score disparity value at each pixel. Steps (1) and (2) are often combined, for a window-based stereo matching approach. Window matching tends to smooth pixels at depth discontinuities. Steps (2) and (3) can be combined if we use a global optimization that tries to model the surface as well. We saw an MRF of a robust prior model for piecewise continuous surface reconstruction in Eq. 4. Inpainting could likely reconstruct structures and holes better by using intuitive processes. A straightforward application of inpainting to steps (2) and (3) above for surface extraction would already be a significant contribution to reconstructing $3 \mathrm{D}$ objects, both the visible and both occluded parts; however, this has not yet been attempted. Additionally, steps (1) and (2) could be made better by inpainting if occluded pixels were aggregated as well, over images and locations in space.

Occlusions present a two-fold problem for multiview stereo. First, they make feature detection problematic, since occlusions can look like structure features (e.g. lines and corners). Reliable features that have correspondence in multiple views are necessary for camera calibration, or projection matrix recovery, without which the entire matching process would be inaccurate. Second, occluded pixels might not be matched even if they are visible in some images, or they might not be visible at all. To date, efforts to combat these effects are specialized and do not tackle the systemic problem of occlusions. Favaro et al. have suggested a way to detect different types of T-junctions [73], which should help produce a more accurate detection of corner features (and incidentally might also help inpainting by detecting occluded lines to be continued). Kang et al. have suggested some techniques to help matching [74]: For area-based matching, they use spatially shiftable, temporally selectable windows. Normally, disparity support aggregation is computed over a window in image space centered at the pixel of interest $p$ in a scheme such as SSSD (Sum of Sum of Squared Differences) where support is summed over all images. However, for pixels near a depth discontinuity, the neighborhood changes in different images, causing a bad match measure. Spatially shiftable windows allow moving the window from being centered at $p$ (near a depth discontinuity) to a location where it does not cross the depth discontinuity. Likewise, for pixels that are occluded in some images but not in the others, temporal selection of windows attempts to heuristically select only windows in images where $p$ is not occluded. Local aggregation techniques also exhibit more difficulties matching textureless regions, so they suggest regressively enlarging window sizes using a criterion of variance on the SSSD measure.

Kang et al. [74] also discussed a global energy minimization for surface reconstruction. Visibility reasoning is used in a similar way to volume reconstruction techniques. The error function is modified by multiplying it by a binary visibility factor that indicates whether a pixel is occluded at some depth:

$$
E(i)=\sum_{k \neq i} \sum_{(x, y) \in S} v(x, y, z, k) g\left(u_{i}(x, y)-H_{i}^{k}(z) u_{k}(x, y)\right),
$$

where $i$ is the reference image we are computing a depth map for, $u$ is the observed image function, $S$ is the domain of $u, z=z(x, y)$ is the depth associated with pixel $(x, y)$, $v$ is the visibility of a depth pixel in image $k, g$ is the truncated quadratic, and $H_{i}^{k}(z)$ is the homography from the plane of depth $z$ in image $k$ to the image plane of image $i$. This process proceeds iteratively by computing the pixel depths using Graph Cuts, and committing a percentage of the best matching depths in the next iterations to assure convergence. Graph Cuts have been shown to be usable for a class of energy functions up to three-parameter clique potentials [75]. A less restrictive symmetric visibility constraint is assumed in [76].

If the surfaces in the image have parametric descriptions, and we try to model them, then our depth matching is made more accurate due to added constraints. Layered images are suggested in [33], where every image is composed of several layers with transparency. The subclass of planar layers is discussed, since it allows easier view transformations via homographies. Each pixel on a layer also has an associated offset depth from the layer. General parametric surfaces might fit scene objects better and add accuracy to surface recovery. This approach supports a generalization of structure and texture inpainting to 3D, where the structures inpainted are layers, and the textures are depth offsets from the layers. Inpainting also implicitly models surface structure better than parametric surfaces.

Szeliski [28] suggested a way to increase matching accuracy in a manner similar to temporal selection by using key-frame views, each with its own depth map. A brightness compatibility term is defined as the SSSD energy given in Eq. 26, with the summation over all key-frames and over the support neighborhood for the key-frames. Two additional terms are used to enforce constraints among the multiple depth maps: a depth smoothness constraint, and a depth compatibility constraint requiring mutual consistency between depths of neighboring key-frames. Rendering is accomplished by warping key-frames to the new viewpoint, and combining them by a volumetric integration technique [67]. This fusion technique is mathematically sound, but might not reconstruct object curves as well as an inpainting approach would do, since the latter would also naturally enforce the depth smoothness con- 
straint. Inpainting should also reduce the need for costly multiple depth maps.

Increasing the number of depth maps to the number of source images, Virtualized Reality was proposed in [27]. A real event is virtualized in a $3 D$ Dome by recording in realtime the images from all cameras positioned in the Dome at regular intervals. A Visible Surface Model (VSM) is constructed for each camera viewpoint using a multi-baseline stereo algorithm. The depth data is tessellated and triangles with large depth differences are considered as a discontinuity and marked as hole triangles. Finally, the mesh is decimated so as not to exceed error conditions. A VSM is rendered via standard graphics techniques such as ZBuffering. It is necessary to combine multiple VSMs to render an image without holes. A reference VSM and two support VSMs are chosen such that they define a bounding triangle for the virtual camera's ray. First the reference VSM is rendered, with its hole triangles rendered into a separate buffer. Then, the two supporting VSMs are rendered only for pixels that are painted in the hole buffer. Finally, holes that are still not covered are interpolated.

Fig. 9 shows the results of a virtual camera moving around a scene of a person swinging a baseball bat. The shading errors around the person occur where a support VSM had to paint over the occlusion holes, and apparently there are shading and geometry differences between two neighboring VSMs, which a depth compatibility constraint could alleviate. A second visual inconsistency that occurs when animating the sequence is a jerky motion when the reference VSM changes. This is again due to inconsistencies in 3D geometry.

A Complete Surface Model (CSM) is suggested to resolve these inconsistencies and enable interaction by generating a consistent 3D surface model. The Curless and Levoy fusion technique is used to integrate all VSMs together [67], and marching cubes tessellation is applied thereafter [71]. Aside from the volume integration method, there is no detection of occlusions, which results in poorer models than those of volume space reconstruction techniques. As noted before, inpainting methods are appropriate here, not only for imposing reasonable surface continuity constraints but also for recovering occluded surfaces for more accurate integration of multiple VSMs.

Jin et al. [65] proposed a multiview stereo approach that can accommodate higher order illumination models. A radiance matrix is established around each point on the current surface and a PDE based evolution is applied aimed at converging when the current shape best estimates the radiance of the true shape. New views are created from projection of the shape and interpolation of the radiance function. This method is less sensitive to shading differences in corresponding pixels values, but its reconstruction is not very precise, and the surface at hole points is in the shape of the original bounding box. Similarly, in [77] a surface evolution is performed such that visibility constraints are maintained. It should prove beneficial to adjust these PDEs to include an inpainting formulation in order to reconstruct surfaces and holes more accurately.

\section{Volumetric Reconstruction Methods}

Volumetric reconstruction methods reconstruct the scene in discretized scene space. This is a simpler approach for supporting visibility reasoning, and so can provide superior matching quality than the depth map methods.

The volumetric stereo reconstruction algorithm given by Szeliski [28] initially performs the same steps of matching and aggregation as in stereo matching. To start with, voxels are filled at locations where the disparity match value is large enough. The volume is then reprojected to each camera's view plane by warping volume depth layers using homography, in a back-to-front order, into the desired view. After reprojection, the pixels in each image that are the same color as the projected volume are marked as visible. This helps determine occluded voxels in the next iteration of matching and voxel filling. An optimization is used to adjust voxel colors and transparencies. This work has an advantage over other volume reconstruction techniques in that it enforces a smoothness constraint instead of a specific regularization criterion that real objects do not possess.

Seitz and Dyer [32] were among the first to suggest a volumetric reconstruction method. Voxel coloring offers a provably consistent photorealistic reconstruction. Photo Integrity of a reconstruction is defined as a reprojection onto the reference images' view planes that reproduces them accurately. The voxel coloring method tries to project the volume in a front-to-back order onto all the images and color only the voxels that are consistent across the images. The pixels that a consistent voxel projects to are marked as recovered, and they are not considered again for color matching. The convex hull of all camera centers is required to be devoid of scene points in order to maintain proper visibility ordering. There can be many consistent scenes for a given set of images. A voxel is considered color invariant with respect to a set of images if for every two consistent scenes in which it appears, its color in both scenes is the same. A voxel coloring of a set of images is therefore the set of all color invariant voxels with respect to the images. The downside of this definition is that the reconstruction builds perturbations volume (cusps) towards the cameras.

The popular space carving theory [4] extends the voxel coloring idea. Space carving attempts to compute a photoconsistent shape from images from arbitrarily placed cameras, as long as scene radiance is locally computable. Here, a multi-sweep approach is used to reason about the photo consistency (as for photo integrity) of each voxel, carving out inconsistent voxels from the volume. The complete reconstruction, called the photo hull, subsumes all photo consistent scenes, and has the same protrusions towards the cameras. It should be noted that a photo hull is an "equivalence class" of all consistent shapes, and it is possible and even advantageous to apply other a-priori constraints such as smoothness, and here inpainting could be of use. In addition to recovering holes and hidden faces, inpainting provides continuation principles that are structure and texture preserving, and thus are significantly better for 

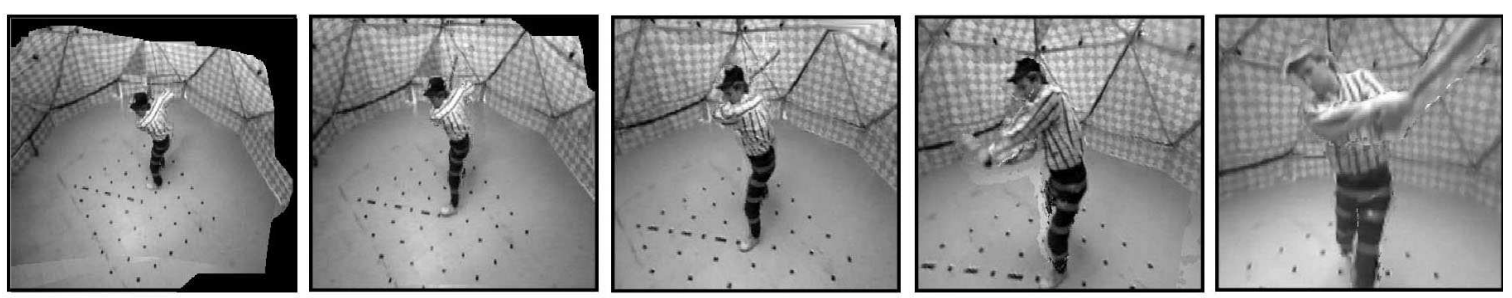

Fig. 9. The virtual camera drops down into a dynamic scene. (From [27]).

reconstruction than simple smoothness assumptions.

For photorealism, the number of voxels required is very large, and so is the number of images potentially required (from a dozen to over a hundred) in order to eliminate false matches. Camera calibration has to be very exact too, here calibrated with sub-pixel precision. The Gargoyle statue rendered in Fig. 10 shows some results of this approach. In the Gargoyle renderings we can see that the surface is not accurate and holes are carved. The inaccuracies are from the bulging effects and insufficient voxel resolution, and holes were carved because there were some inaccuracies due to illumination effects or other noise.

One interesting way to represent volumes so as to make image based rendering fast is by Layered Depth Images (LDI) [33]. A layered depth image is the projective space volume of a single virtual camera, with a few points on each ray added as necessary. The rendering of the LDI from a novel viewpoint is decomposed into incremental warping computations using a scan line approach. Proper visibility ordering is maintained using McMillan's depth ordering algorithm extended to the LDI. The rendering itself is accomplished using splatting [78] with quantized alpha values to facilitate Gaussian type kernels. The LDI provides a fast parallax preserving rendering, but is limited by construction to only close-by views of the scene. However, layered depth images were used for representing the entire 3D structure of objects in Image-Based Objects (IBOs) [79]. We observe that if the LDI is partitioned into 3D surface and texture, and the projection manifold assumes the surface shape, then the points along each ray constitute the texture. Texture and structure inpainting in $3 \mathrm{D}$ can reconstruct the object prior to rendering.

\section{View Dependent Hybrid Methods}

Some improvements in reprojection quality can be accomplished by using view dependent reconstruction for the novel view point. Most methods try to exploit preservation of source image resolution and view dependent shading effects. In Virtualized Reality [27], VSMs were selected that are nearest to the new view.

A visual hull is another reconstruction similar to space carving, but without regard to shading. The foreground in the reference images is called the silhouette, and shooting rays from an image Center Of Projection (COP) through all its foreground pixels creates a cone-like volume with the silhouette cross-section. All the volumes of all the images are combined using Constructive Solid Geometry (CSG) intersection operations. Typically the reconstruc- tion is carried out in volume space, although improvements have been suggested using Delaunay triangulations [80]. An Image-Based Visual Hull (IBVH) construction is suggested in [35] in order to avoid using volume space. For each pixel in the desired view image, its viewing ray is projected onto all reference images, then intersected with the silhouettes and raised back to the $3 \mathrm{D}$ viewing ray for intersection of all such intervals. The shading of the visual hull is performed by selecting the color of the ray in the reference image with closest angle to the desired ray in the new view. This approximates view dependent shading effects.

The method results in a low-detail volume with sharp edges for few cameras, yet it is smoother for surfaces than a volumetric approach. The authors argue that using the visual hull as a surface for texturing, the reconstruction is made to look more realistic than common methods.

An early and successful image-based modeling endeavor deals with architectures in photographs [29]. Initially, the user has to roughly model the scene, which is thereafter matched to input images and adjusted to proper measurements. A view-dependent texture mapping is then applied onto the model, and texture maps of more than one photograph are blended together for smooth transitions. Any pixels still missing after the texture mapping are interpolated. As the 2D texture mapping illusion of depth does not extend well for angles off the original view, and it is unlikely to have a detailed user model, stereo matching is used to extend the 3D structure. Photographs are warped onto the model and projected into the reference plane. Points on the model will have no disparity, while others have a disparity value. The epipolar geometry is preserved as well. Occlusions that are known in the modeled geometry can be handled. The results of this method are very realistic, and show that not only texture mapping over an established geometry improves realism but also the geometry assists with further $3 \mathrm{D}$ reconstruction.

These methods further support our proposal of inpainting structure and texture in 3D. While view-dependent texture inpainting is possible, its effectiveness is unknown. However, a 3D texture in our proposal does not have viewdependent advantages for depth illusion. Instead, it is better to inpaint the texture on the whole to avoid inconsistencies. Additionally, we see that the simple surface resulted from structural inpainting is well suited for texturing. With the additional properties of recovering missing surfaces and realistic continuity principles, inpainting should be an integral part of image-based rendering. 

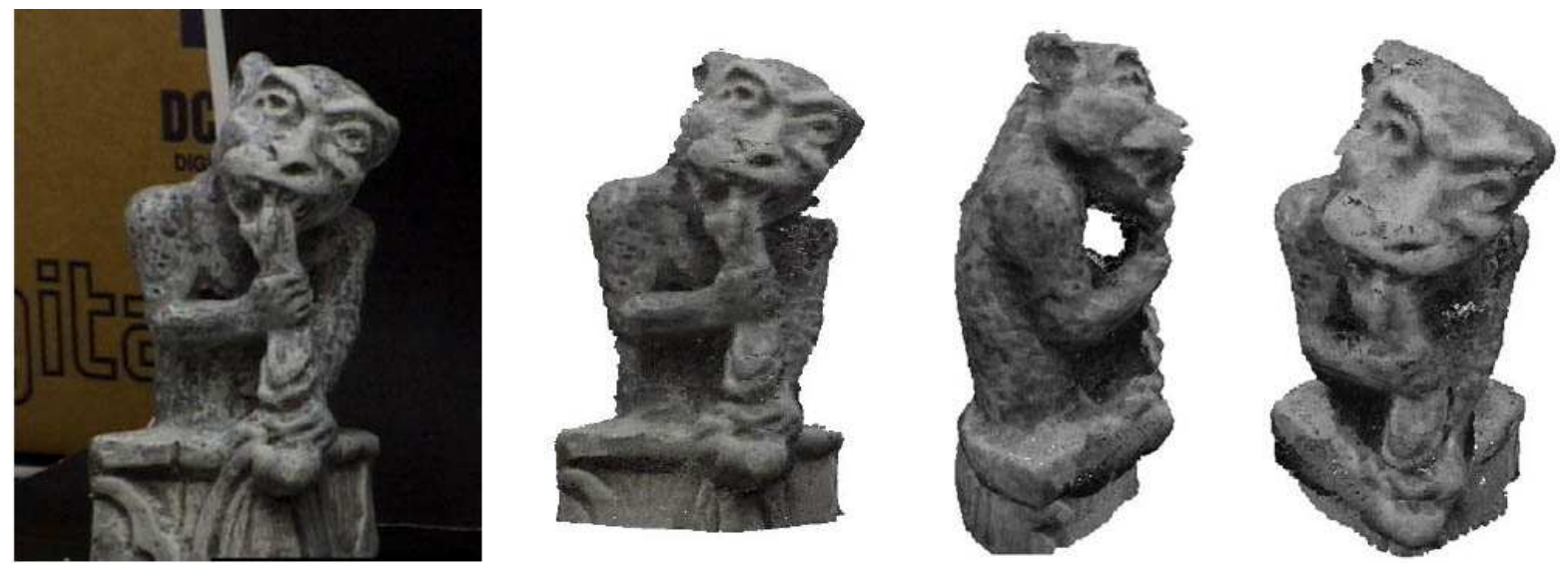

Fig. 10. Gargoyle statue source image and renderings. (From [4]).

\section{Conclusions and Future Work}

This paper provides a review of digital image inpainting, and sets out a case for the necessity of its integration with image-based rendering.

Digital image inpainting has progressed from attempts to disocclude a grey scale object in 2D using straight lines to filling-in of arbitrary holes with texture and shape, and inpainting surfaces in 3D. The current convergence of research in structural inpainting seems to suggest that Euler p-elastica matches the perceived shape best, and is also the mathematical adjunction of previous inpainting methods, while textural inpainting is accomplished by replication from texture exemplars.

Inpainting, however, still has a long way to go before it can be as useful for image-based rendering as envisioned in this review. While it seems obvious that an inpainting functional is tailored for depth surface extraction, there are no current methods that can reliably inpaint large holes in 3D since detail would be lost. Textural inpainting also proceeds either in a statistical fashion or along linear isophotes, and cannot restore complex surfaces. Exploiting statistical principles, we can require symmetry and continuation principles to be upheld. We suggest reconstructing objects by $3 \mathrm{D}$ texture inpainting along smooth structurally inpainted surfaces. This paper presents that approach as both applicable to and necessary for nearly all image-based rendering techniques.

\section{REFERENCES}

[1] R. Hartley and A. Zisserman. Multiple View Geometry in Computer Vision, Second Edition. Cambridge University Press, 2003.

[2] E. Trucco and A. Verri. Introductory Techniques for 3-D Computer Vision. Prentice Hall, 1998.

[3] D. Scharstein and R. Szeliski. A taxonomy and evaluation of dense two-frame stereo correspondence algorithms. Int. J. Comp. Vision, 47(1):7-42, June 2002.

[4] K. S. Kutulakos and S. M. Seitz. A theory of shape by space carving. Int. J. Comp. Vision, Marr Prize Special Issue, 38(3):199-218, 2000.

[5] D. Mumford and J. Shah. Optimal approximations by piecewise smooth functions and associated variational problems. Comm. Pure App. Math., XLII:577-685, 1989.

[6] M. Nitzberg, D. Mumford, and T. Shiota. Filtering, Segmentation and Depth. Lecture Notes in Computer Science, Vol. 662. Springer-Verlag: Berlin, 1993.
[7] M. Bertalmio, G. Sapiro, V. Caselles, and C. Ballester. Image inpainting. Computer Graphics (SIGGRAPH 2000), pages 417424, July 2000.

[8] S. Z. Li. Markov Random Field Modeling in Image Analysis. Springer: Tokyo, 2001.

[9] S. Geman and D. Geman. Stochastic relaxation, gibbs distributions, and the bayesian restoration of images. IEEE Trans. Pattern Analysis and Machine Intelligence (PAMI), 6:721-741, November 1984

[10] S. Masnou and J.-M. Morel. Level lines based disoclussion. Proc. Int. Conf. Image Processing, pages 259-263, 1998.

[11] M. Bertalmio, A. L. Bertozzi, and G. Sapiro. Navier-stokes, fluid dynamics, and image and video inpainting. Proc. IEEE Comp. Vision and Pattern Rec., December 2001.

[12] C. Ballester, M. Bertalmio, V. Caselles, G. Sapiro, and J. Verdera. Filling-in by join interpolation of vector fields and grey levels. IEEE Trans. Image Processing, 10:1200-1211, August 2001.

[13] T. F. Chan, S. H. Kang, and J. Shen. Euler's elastica and curvature based inpaintings. SIAM J. App. Math., 63(2):564$592,2002$.

[14] T. F. Chan and J. Shen. Non-texture inpainting by curvature driven diffusion. J. Visual Comm. Image Rep., 12(4):436-449, 2001.

[15] M. Bertalmio, L. Vesa, G. Sapiro, and S. Osher. Simultaneous structure and texture image inpainting. IEEE Trans. Image Processing, 8:882-889, August 2003.

[16] A. Criminisi, P. Prez, and K. Toyama. Object removal by exemplar-based inpainting. Proc. IEEE Comp. Vision and Pattern Rec., 2:721-728, June 2003.

[17] H. Grossauer. A combined PDE and texture synthesis approach to inpainting. Euro. Conf. Comp. Vision, 2004.

[18] K. A. Patwardhan, G. Sapiro, and M. Bertalmio. Video inpainting of occluding and occluded objects. IEEE Proc. Int. Conf. Image Processing, 2005.

[19] A. Levin, A. Zomet, and Y. Weiss. Learning how to inpaint from global image statistics. Proc. IEEE Int. Conf. Comp. Vision, pages 305-313, October 2003.

[20] S. H. Kang, T. F. Chan, and S. Soatto. Landmark based inpainting from multiple views. TR CAM 02-11, March 2002.

[21] S. Ivekovic and E. Trucco. Dense wide-baseline disparities from conventional stereo for immersive videoconferencing. IEEE Proc. Int. Conf. Pattern Rec., pages 883-897, 2002.

[22] J. Verdera, V. Caselles, M. Bertalmio, and G. Sapiro. Inpainting surface holes. Int. Conf. Image Processing, September 2003.

[23] S. E. Chen and L. Williams. View interpolation for image synthesis. Computer Graphics (SIGGRAPH 1993), 27:279-288, 1993.

[24] M. Levoy and P. Hanrahan. Light field rendering. Computer Graphics (SIGGRAPH 1996), pages 31-42, August 1996.

[25] S. J. Gortler, R. Grzeszczuk, R. Szeliski, and M. F. Cohen. The lumigraph. Computer Graphics (SIGGRAPH 1996), pages 4354, August 1996.

[26] L. McMillan and G. Bishop. Plenoptic modeling: An imagebased rendering system. Computer Graphics (SIGGRAPH 1995), pages 39-46, September 1995. 
[27] P. J. Narayanan, P. W. Rander, and T. Kanade. Constructing virtual worlds using dense stereo. Proc. IEEE Int. Conf. Comp. Vision, pages 3-10, January 1998.

[28] R. Szeliski. Stereo algorithms and representations for imagebased rendering. British Machine Vision Conf., pages 314-328, 1999.

[29] P. E. Debevec, Camillo J. Taylor, and J. Malik. Modeling and rendering architecture from photographs: A hybrid geometryand image-based approach. Computer Graphics (SIGGRAPH 1996), 30(Annual Conference Series):11-20, August 1996.

[30] M. Irani, T. Hassner, and P. Anandan. What does a scene look like from a scene point? Euro. Conf. Comp. Vision, pages 883897, 2002.

[31] J. Sun, H.-Y. Shum, and N.-N. Zheng. Stereo matching using belief propagation. Euro. Conf. Comp. Vision, 2:510-524, 2002.

[32] Steven M. Seitz and Charles R. Dyer. Photorealistic scene reconstruction by voxel coloring. Proc. IEEE Comp. Vision and Pattern Rec., pages 1067-1073, 1997.

[33] J. Shade, S. Gortler, L.-W. He, and R. Szeliski. Layered depth images. Computer Graphics (SIGGRAPH 1998), pages 231242, July 1998.

[34] G. Vogiatzis, P. H. S. Torr, and R. Cipolla. Multi-view stereo via volumetric graph-cuts. Proc. IEEE Comp. Vision and Pattern Rec., 2005.

[35] W. Matusik, C. Buehler, R. Raskar, S. J. Gortler, and L. McMillan. Image-based visual hulls. Computer Graphics (SIGGRAPH 2000), pages 369-374, 2000.

[36] Z. Tauber, Z.-N. Li, and M. S. Drew. Review and preview: Disocclusion by inpainting for image-based rendering. Simon Fraser University, School of Computing Science, TR 2006-04, 2006

[37] D. Ramanan and K. E. Barner. Nonlinear image interpolation through extended permutation filters. Proc. Int. Conf. Image Processing, 2000.

[38] J. Shen. Inpainting and the fundamental problem of image processing. SIAM News, Invited, December 2002.

[39] I. M. Gelfand and S. V. Fomin. Calculus Of Variations. Dover Publications, Inc.: Mineola, New York, 2000

[40] S. Walden. The Ravished Image. St. Martin's Press: New York, 1985

[41] D. King. The Commissar Vanishes. Henry Holt and Company, 1997.

[42] T. K. Shih, R. C. Chang, L. C. Lu, and L. H. Lin. Large block inpainting by color continuation analysis. IEEE Proc. Multimedia Modelling Conf., pages 196 - 202, 2004.

[43] T. K. Shih and R. C. Chang. Digital inpainting survey and multilayer image inpainting algorithms. IEEE Intl. Conf. Info. Tech. and App., 1:15 - 24, 2005.

[44] M. M. Oliveira, B. Bowen, R. McKenna, and Y.-S. Chang. Fast digital inpainting. Proc. Int. Conf. Vis., Imaging and Image Processing, pages 261-266, September 2001.

[45] P. Perona. Orientation diffusions. IEEE Trans. Image Processing, 7(3):457-467, 1999 .

[46] J. Gu, S. Peng, and X. Wang. Digital image inpainting using monte carlo method. IEEE Proc. Int. Conf. Image Processing, 2:961 - 964, 2004.

[47] A. Z. B. Barcelos, M. A. Batista, A. M. Martins, and A. C. Nogueira. Level lines continuation based digital inpainting. IEEE Proc. Brazilian Symp. Comp. Graphics and Image Proc., pages $50-57,2004$

[48] P. Tan, S. Lin, L. Quan, and H.-Y. Shum. Highlight removal by illumination-constraint inpainting. Proc. IEEE Int. Conf. Comp. Vision, pages 826-835, October 2003.

[49] H. Jiang and C. Moloney. Error concealment using a diffusion based method. IEEE Proc. Int. Conf. Image Processing, pages 832-835, 2002

[50] S. Roth and M. J. Black. Fields of experts: A framework for learning image priors. Proc. IEEE Comp. Vision and Pattern Rec., 2:860 - 867, 2005.

[51] L. Ambrosio, N. Fusco, and D. Pallara. Functions of Bounded Variations and Free Discontinuity Problems. Oxford Science Publications. Clarendon Press, 2000.

[52] E. Giusti. Minimal Surfaces and Functions of Bounded Variation. Birkhäuser, Boston, 1984.

[53] T. F. Chan and J. Shen. Mathematical models for local nontexture inpainting. SIAM J. App. Math., 62(3):1019-1043, 2001.

[54] L. Rudin, S. Osher, and E. Fatemi. Nonlinear total variation based noise removal algorithms. Physica D, 60:259-268, 1998.
[55] S. Osher and Sethian J. Fronts propagating with curvature dependent speed: Algorithms based on hamilton-jacobi formulations. J. of Computational Physics, (79):12-49.

[56] D. Tschumperle and R. Deriche. Vector-valued image regularization with PDE's: A common framework for different applications. Proc. IEEE Comp. Vision and Pattern Rec., 1:651-656, 2003.

[57] D. Tschumperle and R. Deriche. Vector-valued image regularization with PDE's: A common framework for different applications. IEEE Trans. Pattern Analysis and Machine Intelligence (PAMI), 27:506-517, 2005

[58] J. S. Yedidia, W. T. Freeman, and Y. Weiss. Constructing free energy approximations and generalized belief propagation algorithms. Mitsubishi Electric Research Labs (MERL), August 2002.

[59] Y. Q. Xu, S. C. Zhu, B. N. Guo, and H. Y. Shum. Asymptotically admissible texture synthesis. Proc. Intl. Workshop on Stat. and Comp. Theories of Vision, 2001.

[60] J.-F. Aujol and A. Chambolle. Dual norms and image decomposition models. Int. J. Comp. Vision, 63(1):85-104, June 2005.

[61] A. Efros and T. K. Leung. Texture synthesis by non-parametric sampling. Proc. IEEE Int. Conf. Comp. Vision, pages 10331038, 1999

[62] L.-Y. Wei and M. Levoy. Order-independent texture synthesis. Computer Science Department, Stanford University, 2002.

[63] Y.-K. Lai, S.-M. Hu, D. X. Gu, and R. R. Martin. Geometry texture synthesis and transfer via geometry images. Proc. ACM Symp. Solid and Physical Modeling (SIGGRAPH), pages 15-26, 2005.

[64] J. Jia and C. K. Tang. Image repairing: Robust image synthesis by adaptive $n$ d tensor voting. Proc. IEEE Comp. Vision and Pattern Rec., 1:643-835, 2003.

[65] H. Jin, S. Soatto, and A. J. Yezzi. Multi-view stereo beyond Lambert. Proc. IEEE Comp. Vision and Pattern Rec., 2003.

[66] O. Faugeras and R. Keriven. Variational principles, surface evolution, PDE's, level set methods, and the stereo problem. IEEE Trans. Image Processing, 7(3):336-344, March 1998.

[67] B. Curless and M. Levoy. A volumetric method for building complex models from range images. Computer Graphics (SIGGRAPH 1996), pages 303-312, 1996.

[68] Y. Duan, Liu Yang, H. Qin, and D. Samaras. Shape reconstruction from 3D and 2D data using PDE-based deformable surfaces. Euro. Conf. Comp. Vision, 2004.

[69] F. Han and S.-C. Zhu. Bayesian reconstruction of 3D shapes and scenes from a single image. Proc. IEEE Int. Conf. Comp. Vision, 2003.

[70] B. Lichtenbelt, R. Crane, and S. Nagvi. Introduction to Volume Rendering. (Hewlett-Packard Professional Books). Prentice Hall, 1998.

[71] W. E. Lorensen and H. E. Cline. Marching cubes: A high resolution 3D surface construction algorithm. Computer Graphics (SIGGRAPH 1987), pages 163-169, July 1987.

[72] J. D. Foley, A. van Dam, S. K. Feiner, and J. F. Hughes. Computer Graphics: Principles and Practice in $C$, second edition. Addison-Wesley Professional, 1995.

[73] P. Favaro, A. Duci, Y. Ma, and S. Soatto. On exploring occlusions in multiple view geometry. Proc. IEEE Int. Conf. Comp. Vision, October 2003.

[74] S. B. Kang, R. Szeliski, and J. Chai. Handling occlusions in dense multi-view stereo. Proc. IEEE Comp. Vision and Pattern Rec., 4:921-924, Aug 2004.

[75] V. Kolmogorov and R. Zabih. What energy functions can be minimized via graph cuts? Proc. Euro. Conf. Comp. Vision, III:65-81, May 2002.

[76] J. Sun, Y. Li, S. B. Kang, and H.-Y. Shum. Symmetric stereo matching for occlusion handling. Proc. IEEE Comp. Vision and Pattern Rec., 2005.

[77] J.E. Solem, F. Kahl, and A. Heyden. Visibility constrained surface evolution. Proc. IEEE Comp. Vision and Pattern Rec., 2005.

[78] K. Mueller, T. Möller, and R. Crawfis. Splatting without the blur. IEEE. Visualization, pages 363-370, 1999.

[79] M. M. Oliveira and G. Bishop. Image-based objects. Proc. ACM Symposium Interactive 3D Graphics, April 1999.

[80] E. Boyer and J.-S. Franco. A hybrid approach for computing visual hulls of complex objects. Proc. IEEE Comp. Vision and Pattern Rec., 1:695-701, 2003. 